\title{
Reaching into the $N=40$ Island of Inversion with Nucleon Removal Reactions
}

\author{
Alexandra Gade ${ }^{1,2}$ (D) \\ 1 FRIB Laboratory, Michigan State University, East Lansing, MI 48824, USA; gade@nscl.msu.edu \\ 2 Department of Physics and Astronomy, Michigan State University, East Lansing, MI 48824, USA
}

Citation: Gade, A Reaching into the $N=40$ Island of Inversion with

Nucleon Removal Reactions. Physics 2021, 3, 1226-1236. https://doi.org/ $10.3390 /$ physics 3040077

Received: 29 October 2021

Accepted: 2 December 2021

Published: 8 December 2021

Publisher's Note: MDPI stays neutral with regard to jurisdictional claims in published maps and institutional affiliations.

Copyright: (C) 2021 by the author. Licensee MDPI, Basel, Switzerland. This article is an open access article distributed under the terms and conditions of the Creative Commons Attribution (CC BY) license (https:// creativecommons.org/licenses/by/ $4.0 /)$.

\begin{abstract}
One ambitious goal of nuclear physics is a predictive model of all nuclei, including the ones at the fringes of the nuclear chart which may remain out of experimental reach. Certain regions of the chart are providing formidable testing grounds for nuclear models in this quest as they display rapid structural evolution from one nucleus to another or phenomena such as shape coexistence. Observables measured for such nuclei can confirm or refute our understanding of the driving forces of the evolution of nuclear structure away from stability where textbook nuclear physics has been proven to not apply anymore. This paper briefly reviews the emerging picture for the very neutron-rich $\mathrm{Fe}, \mathrm{Cr}$, and Ti isotopes within the so-called $N=40$ island of inversion as obtained with nucleon knockout reactions. These have provided some of the most detailed nuclear spectroscopy in very neutron-rich nuclei produced at rare-isotope facilities. The results indicate that our current understanding, as encoded in large-scale shell-model calculations, appears correct with exciting predictions for the $N=40$ island of inversion left to be proven in the experiment. A bright future emerges with predictions of continued shell evolution and shape coexistence out to neutron number $N=50$, below ${ }^{78} \mathrm{Ni}$ on the chart of nuclei.
\end{abstract}

Keywords: rare isotopes; shell evolution; $N=40$ island of inversion; knockout reactions

\section{Introduction}

One of the challenging goals of the field of nuclear structure physics is to model atomic nuclei, including their properties and their reactions-rooted in the fundamental forces at play between protons and neutrons - with predictive power also for the shortestlived nuclear species located near the driplines of the chart. With this ultimate vision to extrapolate towards the most neutron-rich nuclei that may elude experimental study in the near future, much can be gleaned from nuclei in regions that display the effects of structural evolution away from the valley of stability and so offer a window into the driving forces of structural change and our understanding of it [1-3]. Specifically, the complex interplay between single-particle and collective degrees of freedom can provide exciting experimental challenges and demanding theoretical benchmarks.

The region of rapid structural change of interest in this review is the so-called " $N=40$ island of inversion" [4,5], where the neutron-rich Fe and $\mathrm{Cr}$ nuclei around neutron number 40 become the most deformed in the region. In nuclear models, this is theorized to be caused by the strong quadrupole-quadrupole interaction producing a nuclear shape transition in which highly-correlated many-particle-many-hole configurations become energetically more favored than the normal-order (spherical) ones [4]. Such islands of inversion are characterized by rapid structural changes and shape coexistence [5,6], providing insight into nuclear structure physics far from stability [7]. Large-scale shell-model calculations with the LNPS (Lenzi-Nowacki-Poves-Sieja) effective interaction [4] in the full $f p$ shell for protons and the $f_{5 / 2}, p_{3 / 2}, p_{1 / 2}, g_{9 / 2}$, and $d_{5 / 2}$ orbitals for neutrons have confirmed the picture described above, with many successful predictions that preceded experimental results [3]. 
A recent prediction extends this island of inversion to $N=50$ [5] and includes nuclei that will only be reached at next-generation rare-isotope beam facilities. This exciting prospect of extending the island towards the magic neutron number $N=50$ is based on extrapolations of calculations using the LNPS shell-model effective interaction and its monopole drifts $[4,5]$. These prediction together with advances in experimentation continue to push the field forward on the journey to the $N=50$ island of inversion.

The furthest experimental reach into the $\mathrm{Fe}, \mathrm{Cr}$, and $\mathrm{Ti}$ isotopes has been afforded by inverse-kinematics nucleon removal studies induced by fast rare-isotope projectile beams [8,9] to probe the nuclei of interest via in-beam $\gamma$-ray spectroscopy [10]. Often, such reactions provide the first glimpse of the excitation level scheme [11] and, in some cases, the direct character of such reactions is used to conclude on wave function overlaps within the shell-model framework $[8,9]$. This paper reviews the recent results for the very neutron-rich nuclei ${ }^{66,68,70,72} \mathrm{Fe},{ }^{64,66} \mathrm{Cr}$, and ${ }^{60,62} \mathrm{Ti}$, all located near the center of the $N=40$ island of inversion or already on the path to $N=50$, obtained with such experimental approaches which have provided pioneering information the furthest away from the line of stability; see Figure 1.

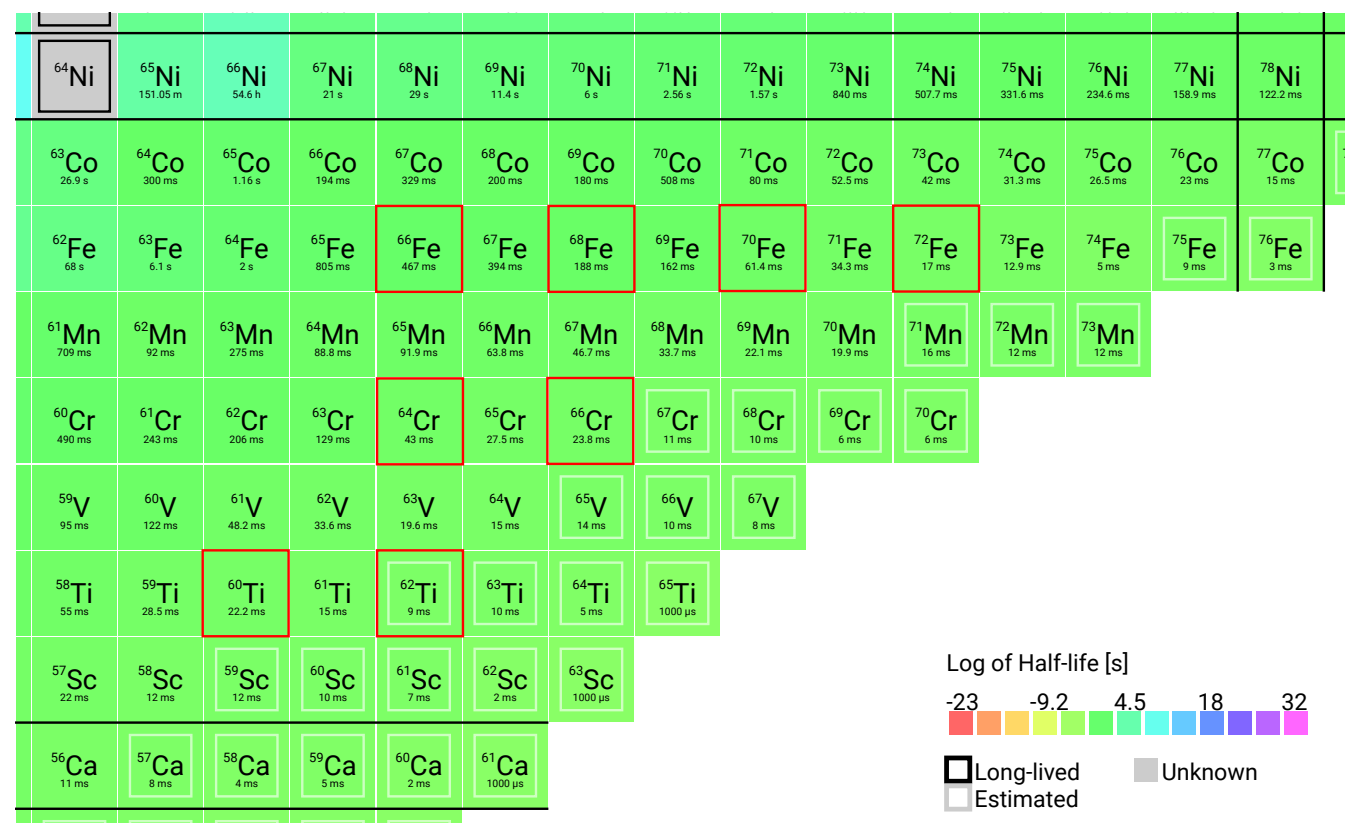

Figure 1. Portion of the nuclear chart that shows the $N=40$ island of inversion. Nuclei discussed in this review are highlighted with a red outline. This chart was generated with [12] (half-life color coding based on NuBase2020 and corresponding extrapolations).

\section{Experimental Approaches}

Experimental techniques aimed at tracking the changes in the structure of nuclei are multi-pronged. They include measurements of ground-state properties such as masses, radii, $\beta$-decay properties, and electromagnetic moments as well as the study of properties of bound and unbound excited states. One way of probing specific nuclear structure aspects in quantitative ways is the use of nuclear reactions that selectively probe a specific degree of freedom. Inelastic scattering of nuclei, including Coulomb excitation, has long been used to probe nuclear collectivity, characterized by the coherent motion of several protons and neutrons. The single-particle degree of freedom, on the other hand, is commonly associated with the single-particle composition of the many-body wave function in a shell-model picture. Such single-particle properties can be studied rather selectively by using direct reactions that add or remove one or a few nucleons from the nucleus of interest. Intriguing possibilities now arise in the above mentioned islands of inversion, where the telltale onset of collectivity and the underlying migration of single-particle levels can be tracked to provide a consistent picture. 
At in-flight rare-isotope facilities, short-lived nuclei away from stability can be efficiently produced by fragmentation (or fission) of stable, primary beams impinging upon stable targets at high beam energy. The resulting secondary beams of rare isotopes are then available for experiments at velocities typically exceeding a $v / c$ of $30 \%$, where $c$ is the speed of light. Well-established experimental techniques used for decades to study stable nuclei are not readily applicable in inverse kinematics and at the low beam rates encountered for the shortest-lived nuclear species. Instead, powerful new experimental approaches have been developed to enable in-beam nuclear spectroscopy studies of fast rare-isotope beams with intensities that are several orders of magnitude less than needed for typical low-energy techniques.

The intensities of rare-isotope beams are lower than stable-beam rates by several orders of magnitude. However, the experimental approach of in-beam $\gamma$-ray spectroscopy compensates for the reduced intensities by enabling thick reaction targets, due to the high beam velocity, and realizing measurements with luminosities comparable to stable-beam experiments but at beam rates of up to a factor of $10^{4}$ less. Reactions such as nucleon removal are induced in thick reaction targets (several hundred $\mathrm{mg} / \mathrm{cm}^{2}$ to g/ $\mathrm{cm}^{2}$ ) and with the detection of $\gamma$ rays for the identification of the reaction residue's final state [10]. Since the residue's $\gamma$-ray emission occurs in flight, the $\gamma$-ray detection systems have to be granular or position-sensitive to allow for an angle-dependent event-by-event reconstruction of the Doppler-shifted $\gamma$-ray energies into the rest frame of the emitter. The choice of the target material depends on the desired reaction; one- and two-nucleon knockout reactions [8] are often induced by light targets, for example ${ }^{9} \mathrm{Be}$ or ${ }^{12} \mathrm{C}$, while quasi-free scattering of the $(p, 2 p)$ or $(p, p n)$ type are nowadays performed with MINOS, an extended liquid hydrogen target that allows reaction vertex-reconstruction and tracking following the concept of a time projection chamber [13]. The projectile-like reaction residues exiting the target has to be identified with magnetic spectrographs or advanced detector systems to cleanly select the reaction channel of interest. In-beam $\gamma$-ray spectroscopy programs with fast beams are pursued at a number of fragmentation facilities around the world, while the work using nucleon removal reactions in the $N=40$ region has been performed largely at NSCL [14] in the US and RIBF/RIKEN [15] in Japan with the GRETINA [16,17] and DALI2 [18] arrays for $\gamma$-ray spectroscopy, respectively. A sketch of the experimental scheme is shown in Figure 2.

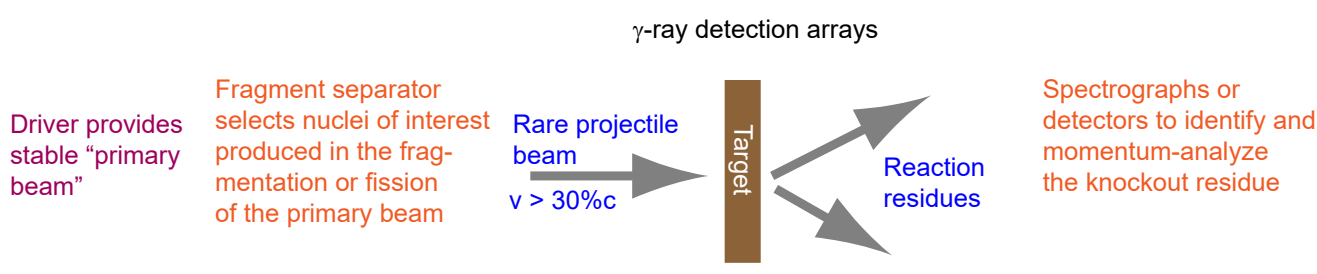

Figure 2. Experimental scheme for inverse-kinematics nucleon knockout reactions at rare-isotope beam facilities that provide fast beams of rare isotopes via projectile fragmentation or fission with velocities, $v$, exceeding $30 \%$ of the speed of light, $c$.

For ${ }^{9} \mathrm{Be}$ - or ${ }^{12} \mathrm{C}$-induced one-nucleon knockout reactions, the exit channel of interest is one where-in a single step-one proton or neutron is removed from the fast rareisotope beam and the projectile-like residue with one less nucleon survives in a bound final state. This channel is characterized by swift, surface-grazing collisions of the projectile and the target nuclei. From a large body of experiments performed at energies from $50 \mathrm{MeV} /$ nucleon to more than $1 \mathrm{GeV} /$ nucleon at (rare-isotope) facilities around the world, it has been established that, with large cross sections, the dominant single-hole states relative to the projectile ground state are populated in the projectile-like reaction residue, unambiguously demonstrating the unmatched sensitivity to the single-particle degree of freedom. The residue parallel momentum distributions encode in their shape and width the information of the orbital angular momentum $\ell$ and separation energy of the removed 
nucleon [8]. The cross section of the selectively populated single-hole configurations scale with the respective spectroscopic factor or wave-function overlap in a shell-model picture. Statistical descriptions of these reactions will not capture these features. Comparisons of such one-nucleon removal data with nuclear structure calculations have been enabled by a direct reaction model $[8,19,20]$ that uses the sudden (short interaction time) and the spectator-core approximation to many-body eikonal (forward-scattering) theory [19] with a detailed prescription provided in [21]. The single-particle nuclear structure information then enters the calculations through spectroscopic factors, or wave-function overlaps, that scale the calculated cross sections for the removal of one nucleon from the corresponding orbital. With that, the measured knockout cross sections can serve as formidable probes of shell-model interactions on the quest to identify the single-particle makeup of the projectile ground state and the residue final states [8,22].

It has been shown also that two-proton and two-neutron removal from neutron-rich and neutron-deficient projectiles, respectively, also proceed as direct reactions [23-25]. By combining eikonal reaction dynamics, that assumes a sudden single-step removal of two nucleons and shell-model calculations of the two-nucleon amplitudes (TNAs), the cross sections for two-nucleon knockout from the parent-nucleus ground state to each of the final states in the daughter nucleus can be calculated [26]. Also, it was shown that the shape of the parallel momentum distribution of the two-nucleon knockout residues depends strongly on the total angular momentum of the two removed nucleons, allowing spin values to be assigned to populated final states [27-29]. One step further, it was proposed and confirmed that since the two-nucleon overlaps contain components with different values of the total orbital angular momentum, information beyond the total angular momentum can be probed. This opens up the possibility to uniquely explore this composition and couplings within the wave functions of rare isotopes [30,31].

More recently, quasi-free $(p, 2 p)$ and $(p, p n)$ reactions, extensively used in normal kinematics with stable targets, have been successfully adapted for inverse-kinematics studies of rare-isotope beams on proton targets [9]. Just as the heavy-ion-induced knockout reactions sketched above, the proton-induced knockout reaction selectively probes the single-particle structure of the nucleus of interest. Also, the shape of the momentum distribution of the knockout residue is connected to the momentum distribution of the knocked-out nucleon. Protons are a penetrating probe that interrogate the nuclear interior, and their rescattering inside the nucleus has to be understood and modeled [9]. In heavyion induced knockout, the orbital radii need to be modeled precisely due to their surface localization [21]. This experimental approach has been used recently at RIBF/RIKEN for measurements reviewed here. Various reaction models have been developed and their consistency remains a challenge for the future [32]. The different nucleon removal reactions were described and confronted with each other recently and extensive details on sensitivities and model dependencies can be found in reference [32].

\section{The Fe Isotopic Chain}

The first in-beam nuclear spectroscopy of ${ }^{66} \mathrm{Fe}$ and ${ }^{68} \mathrm{Fe}$ was published in 2008 from a measurement performed at NSCL where these two Fe isotopes with 40 and 42 neutrons were populated each in ${ }^{9} \mathrm{Be}$-induced one- and two-proton knockout reactions, using the laboratory's S800 spectrograph for particle identification and SeGA for in-beam $\gamma$-ray spectroscopy [33]. For ${ }^{66} \mathrm{Fe}$, in addition to the tentative $2_{1}^{+} \rightarrow 0_{1}^{+}$and $4_{1}^{+} \rightarrow 2_{1}^{+}$transitions reported earlier from $\beta$ decay [34], a 957(10) $\mathrm{keV} \gamma$-ray was observed in both reactions, while a 1310(15) keV transition was only seen in the one-proton knockout. Within a simple two-proton knockout picture, for two protons removed from the $f_{7 / 2}$ orbital, one would expect to populate states in ${ }^{66} \mathrm{Fe}$ with spin-parity $6^{+}, 4^{+}$, and $2^{+}$, suggesting that the $957-$ $\mathrm{keV}$ line depopulates the $6^{+}$state of ${ }^{66} \mathrm{Fe}$. Subsequent $\beta$-decay work limited to lower-spin states seems to suggest that the $1310-\mathrm{keV}$ transition could originate from the second $2^{+}$ level and feeds the first $2^{+}$state [35]. This first in-beam work predates the publication of the LNPS effective interaction [4] and it is interesting to explore the suggestion that 
the $6_{1}^{+}$state was observed. Kotila and Lenzi [36] discuss collective phenomena in Fe and $\mathrm{Cr}$ and show that the $6_{1}^{+}$level predicted by the LNPS calculations agrees with the tentatively assigned $\left(6^{+}\right)$state proposed by Adrich et al. in ${ }^{66} \mathrm{Fe}$ [36]. Subsequent inbeam spectroscopy work performed at NSCL explored the $2_{1}^{+}$and $4_{1}^{+}$states of ${ }^{66} \mathrm{Fe}$ in ${ }^{9}$ Be-induced inelastic scattering [37] and the quadrupole collectivity in intermediate-energy Coulomb excitation [38] and excited-state lifetime measurements [39].

Beyond $N=40$, in ${ }^{68} \mathrm{Fe}$, the first observation of $\gamma$-ray transitions was reported in [33] from ${ }^{9} \mathrm{Be}$-induced one- and two-proton removal reactions with proposed $2_{1}^{+} \rightarrow 0_{1}^{+}$and $4_{1}^{+} \rightarrow 2_{1}^{+}$decays, later supported by intermediate-energy Coulomb excitation measurements [38] as well as $\beta$ decay [40]. It turned out that the energy of the first $2^{+}$state in ${ }^{68} \mathrm{Fe}$ is lower than in ${ }^{66} \mathrm{Fe}$, indicating that the maximum collectivity is assumed beyond $N=40$. Taking the $4_{1}^{+}$assignment at face value, the $R_{4 / 2}$ energy ratio increases as well. The shell model calculations with the LNPS effective interaction are in good agreement with the energies and transition strengths, lending even more confidence that the shell evolution past $N=40$ is captured by the incorporated driving forces [4]. Excited states beyond the tentatively assigned yrast $2^{+}$and $4^{+}$remained elusive until a $\beta$-decay study [41], where a number of low-spin states were proposed. Two candidates for the $6_{1}^{+}$state just emerged recently from a ${ }^{9} \mathrm{Be}$-induced charge-exchange reaction on ${ }^{68} \mathrm{Co}$ projectiles in the $\left(7^{-}\right)$ground state and a low-spin isomer [42]. Governed by the charge-exchange selection rules, access to never-before observed states was provided, predominantly higher-spin states [42]. The calculations with the LNPS effective interaction show good agreement with the energies of the candidate yrast states up to the suggested candidate $6^{+}$levels [42]. This reaction mechanism holds great promise to reach beyond the selectivity of knockout reactions and $\beta$ decay, depending on the spin and parity of the projectile initial state.

At $N=44$, spectroscopy of ${ }^{70} \mathrm{Fe}$ became first possible in 2015 at the RIBF facility at RIKEN using a $(p, 2 p)$ reaction with the MINOS hydrogen target [13] and the DALI2 scintillator array [43] and in the same year with $\beta$ decay at RIKEN [41]. Two transitions were consistently identified in both measurements and proposed to correspond to the $2_{1}^{+} \rightarrow 0_{1}^{+}$ and $4_{1}^{+} \rightarrow 2_{1}^{+}$decays, establishing the corresponding states. It took until 2019 to get beyond the yrast $4^{+}$state and identify a transition on top of the $4^{+}$level in a ${ }^{9} \mathrm{Be}$-induced one-proton knockout measurement performed at NSCL with GRETINA and the S800 spectrograph [44]. The measured and calculated partial one-proton removal cross sections were confronted and showed, at first glance, a striking disagreement with high-lying states populated more strongly than the yrast states observed in the measurement. The emerging picture is one that is not unlike the Pandemonium in $\beta$ decay [45], where indiscernible feeding from a multitude of higher-lying states funnels intensity into low-lying states which then appear prominent albeit carrying little direct feeding. This demonstrates that, while one-proton removal is a powerful experimental probe to reach nuclei more neutron-rich than the projectile, the collectivity prevalent in this region of the nuclear chart can lead to fragmentation of the single-particle strength which may then be thinly spread over many states in the reaction residue, leading to a Pandemonium-like feeding scheme when $\gamma$-ray spectroscopy is used [44].

The most neutron-rich Fe isotope with spectroscopic information is ${ }^{72} \mathrm{Fe}$, studied at RIBF/RIKEN in the same experiment and with the same approach as ${ }^{70} \mathrm{Fe}$ [43] and two $\gamma$ rays were observed and proposed to correspond to the $2_{1}^{+} \rightarrow 0_{1}^{+}$and $4_{1}^{+} \rightarrow 2_{1}^{+}$ transitions, establishing the corresponding states. It will likely take a next-generation rare-isotope facility to move beyond ${ }^{72} \mathrm{Fe}$ with nuclear spectroscopy. A peculiar picture emerges where starting at $N=40$, the evolution of the $2_{1}^{+}$and $4_{1}^{+}$excitation energies largely stays flat, as shown in Figure 3. Across the Fe isotopic chain, the LNPS shellmodel calculations, using the slightly modified LNPS-m effective interaction, reproduce the measured excitation energies. 


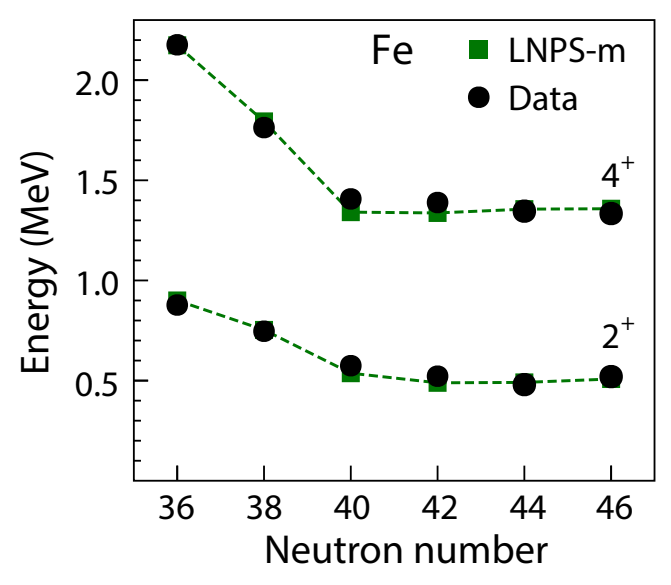

Figure 3. Evolution of the yrast $2^{+}$and $4^{+}$states in the Fe isotopic chain from $N=36$ to 46 , the most neutron-rich Fe isotope with spectroscopic information. The data is confronted with the results of LNPS-m (modified Lenzi-Nowacki-Poves-Sieja) shell-model calculations from reference [43]. LNPS$\mathrm{m}$ is a slightly modified version of the original LNPS interaction as detailed in [43]. The calculations reproduce the signature drop in excitation energy at $N=40$, corresponding to an onset of collectivity, and the subsequent flat evolution.

\section{The Cr Isotopes}

In the heart of the $N=40$ island of inversion, the nucleus ${ }^{64} \mathrm{Cr}$ eluded spectroscopy until 2010. In fact, Adrich et al. attempted to populate ${ }^{64} \mathrm{Cr}$ in the two-proton removal from ${ }^{66} \mathrm{Fe}$. This measurement failed as the cross section turned out to be only $0.13(5) \mathrm{mb}$, an order of magnitude smaller than the cross section leading from ${ }^{68} \mathrm{Ni}$ to ${ }^{66} \mathrm{Fe}$ along $N=40$ [33]. The conclusion at the time was that the cross section is small due to a structural mismatch between the ${ }^{66} \mathrm{Fe}$ ground state and the bound states of ${ }^{64} \mathrm{Cr}$. This early idea was partially supported in 2010 through the LNPS effective interaction which predicts significant differences in the neutron $2 \mathrm{p} 2 \mathrm{~h}$ and $6 \mathrm{p} 6 \mathrm{~h}$ content in the ground states of ${ }^{66} \mathrm{Fe}$ and ${ }^{64} \mathrm{Cr}$, hinting indeed at a potentially reduced overlap of the neutron wave functions [4]. The first spectroscopy of ${ }^{64} \mathrm{Cr}$ was then accomplished via ${ }^{9} \mathrm{Be}$-induced inelastic scattering at NSCL where candidates for the $2_{1}^{+} \rightarrow 0_{1}^{+}$and $4_{1}^{+} \rightarrow 2_{1}^{+}$transitions were identified. While published in the same year, this data did not enter the development of the LNPS effective interaction and so the close match between experiment and calculation can be viewed as a stunningly successful prediction [4]. The $2_{1}^{+}$state and the energy of the candidate $4_{1}^{+}$level were since confirmed in intermediate-energy Coulomb excitation [38] and $\beta$ decay [46], respectively. The first one- and two-proton knockout study into ${ }^{64} \mathrm{Cr}$, using GRETINA and the S800, revealed a high $\gamma$-ray transition density, indicative of a rather complex and dense level scheme [47]. A quantitative knockout study was not possible as the knockout reaction channels may have contained small contaminations from ${ }^{64} \mathrm{Cr}$ populated in fragmentation of other projectiles in the cocktail beam [47]. A study of the $B(E 2)$ transition strength predicted by the LNPS shell-model calculations revealed several very interesting collective band structures, resembling a gamma and beta band but with deviations from the textbook expectations for such structures [47]. These proposed bands are barely linked via E2 transitions. Identifying these predicted collective structures in measurements has to remain a challenge for future studies and next-generation rare-isotope facilities where these states can be accessed with reactions at low beam energies such as deep-inelastic scattering [47].

The most neutron-rich $\mathrm{Cr}$ isotope with spectroscopic information is ${ }^{66} \mathrm{Cr}$ studied at RIBF/RIKEN with $\gamma$-ray spectroscopy following a $(p, 2 p)$ reaction [43]. Candidate transitions for the $2_{1}^{+} \rightarrow 0_{1}^{+}$and $4_{1}^{+} \rightarrow 2_{1}^{+}$decays were proposed, in agreement with slightly modified LNPS shell-model calculations, termed LNPS-m in [43]. The Cr isotopes mirror the observations for the Fe isotopic chain, with a rather flat evolution of the $2_{1}^{+}$and $4_{1}^{+}$energies, but starting at $N=38$ already instead of at $N=40$ as for the Fe chain; see Figure 4 . 


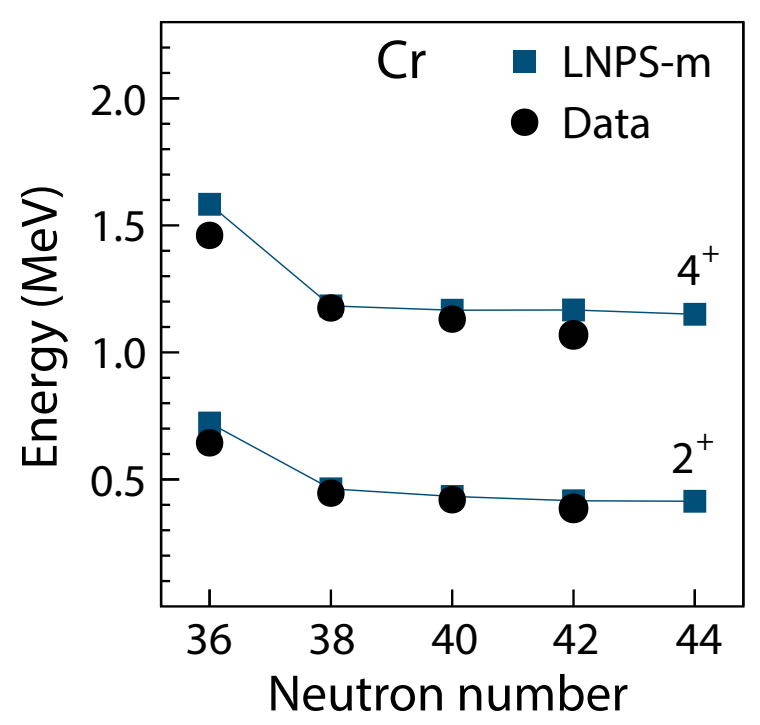

Figure 4. Evolution of the yrast $2^{+}$and $4^{+}$states in the $\mathrm{Cr}$ isotopic chain from $N=36$ to 44 , the most neutron-rich $\mathrm{Cr}$ isotope with spectroscopic information. The data is confronted with the results of LNPS-m shell-model calculations from reference [43]. LNPS-m is a slightly modified version of the original LNPS interaction as discussed in [43]. The calculations reproduce the signature drop in excitation energy at $N=38$, corresponding to an onset of collectivity, and the subsequent flat evolution. Note that the onset of collectivity in $\mathrm{Cr}$ sets in already at $N=38$, unlike for the Fe isotopes.

\section{The Ti Isotopic Chain}

The $N=38 \mathrm{Ti}$ isotope ${ }^{60} \mathrm{Ti}$ was studied in the ${ }^{9} \mathrm{Be}$-induced one-proton knockout at NSCL using GRETINA at the S800 spectrograph, providing the first spectroscopy of this nucleus [48]. One $\gamma$-ray peak was observed which was argued to be a doublet of two transitions corresponding to the $2_{1}^{+} \rightarrow 0_{1}^{+}$transition and perhaps the decay of the $4_{1}^{+}$level. This measurement exploited the knockout reaction mechanism and compared calculated and measured partial cross sections [48]. The comparison supported the suggestion of a doublet as well as the spin assignments for the candidate states and the expectation for the inclusive cross section. This analysis provided a unique benchmark for the LNPS effective interaction that goes beyond excitation energies and includes wave-function overlaps, at the time the closest to ${ }^{60} \mathrm{Ca}$ as possible.

At $N=40,{ }^{62} \mathrm{Ti}$ was accessed with $\gamma$-ray spectroscopy only recently, using a $(p, 2 p)$ reaction with the MINOS target and the DALI2 scintillator array at SAMURAI [49]. Candidate $\gamma$-ray decays attributed to the $2_{1}^{+} \rightarrow 0_{1}^{+}$and $4_{1}^{+} \rightarrow 2_{1}^{+}$transitions were proposed. As in the work on ${ }^{60} \mathrm{Ti}$ [48], also for ${ }^{62} \mathrm{Ti}$ the direct nature of the reaction was exploited, comparing measured and calculated partial cross sections that probe the wave-function overlaps between projectile ground state and knockout residue final states. $\mathrm{A}^{63} \mathrm{~V}$ ground-state spin assignment $J=3 / 2^{-}$was found the most likely given the calculated cross section distributions for the other alternatives [49]. Along the $N=40$ isotone line, ${ }^{62} \mathrm{Ti}$ is the last extrapolation point towards the elusive ${ }^{60} \mathrm{Ca}$ (see Figure 5), which was proven to exist only recently with implications for the dripline in the Ca isotopic chain [50]. 


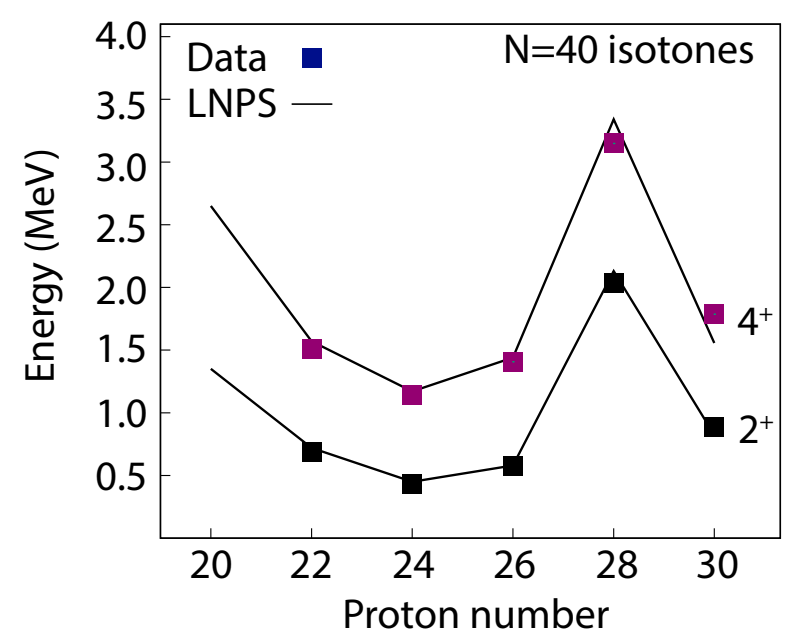

Figure 5. Evolution of the $2^{+}$and $4^{+}$energies in the $N=40$ isotones from Ca to $\mathrm{Zn}$ as predicted by LNPS shell-model calculations presented in reference [49] in comparison to data, where ${ }^{62} \mathrm{Ti}$ is the most neutron-rich in the chain. The excellent agreement lends confidence in the prediction for the elusive nucleus ${ }^{60} \mathrm{Ca}$ which was only recently identified [50].

\section{Complementary Descriptions of the Region}

While this review focuses on the shell-model description of the nuclei above, the reader is referred to an interesting discussion of the $\mathrm{Cr}$ and Fe isotopic chains in the framework of the proton neutron interacting boson model (IBM-2) by Kotila and Lenzi [36]. Among the discussed collective observables, for example, the measured as well calculated energy ratios $R_{4 / 2}$ and $R_{6 / 4}$ within the shell-model and the IBM-2 are examined from $N=30-40$ [36]. Complementary to the effective-interaction shell model and the IBM, Coraggio et al. [51] performed pioneering realistic shell-model calculations starting from a low-momentum potential derived from the high-precision CD-Bonn free nucleon-nucleon interaction. The energies of the first $2^{+}$states and $B(E 2)$ strengths are calculated inside the $N=40$ island of inversion and the best agreement is reached with the largest possible model space [51]. These calculations were extended for ${ }^{68,70} \mathrm{Fe}$ and confronted with experiment in [41]. The level structures of odd- $\mathrm{Z}^{63,65,67} \mathrm{Mn}$ isotopes located on the nuclear chart just between the collective $\mathrm{Cr}$ and Fe isotopic chains were shown to be consistent with strongly coupled rotational bands built on a state with $K$ quantum number $K=5 / 2$ [52], providing yet another means to characterize the collectivity that has become a hallmark of the region.

\section{Summary and Conclusions}

The $N=40$ island of inversion, centered on ${ }^{64} \mathrm{Cr}$, has enjoyed intense attention from experimentalists and theorists alike. Experimental efforts at NSCL/MSU and RIBF/RIKEN have pushed the frontiers of spectroscopy by utilizing proton removal reactions which always lead to reaction residues more neutron-rich than the projectile. Technological advances, such as GRETINA at NSCL and MINOS at RIBF, allowed $\gamma$-ray spectroscopy, for the first time, at $N=46, N=42$ and $N=40$ in the $\mathrm{Fe}, \mathrm{Cr}$, and Ti isotopic chains, respectively. On the theory side, the LNPS effective shell-model interaction continues to demonstrate not just the capability to reproduce the data but also to predict some of it. This close collaboration between experiment and theory has led to continuous refinements of the LNPS effective interaction and, in turn, motivated cutting-edge measurements at rare-isotope facilities around the world. Particularly exciting are predictions of an $N=50$ island of inversion which rely on the monopole drifts from the LNPS interaction. This unmatched success of this effective interaction at $N=40$ and beyond now lends confidence to these extrapolation and promises an exciting future for experiments at next generation rare-isotope facilities needed to reach these outskirts of the nuclear chart. In the $N=40$ island of inversion itself, open challenges for experiment remain with respect to shape and configuration coexistence as predicted to be manifested in quadrupole-collective band 
structures in ${ }^{62,64} \mathrm{Cr}$, for example. Also the study of such higher-lying off-yrast states has to remain a challenge for upcoming rare-isotope facilities. The future is exciting with experimental and theoretical advances in lockstep pushing the field forward to conquer the $N=50$ island of inversion and fully characterize the $N=40$ one. For this, more exotic fast-beam reactions such as the HI-induced charge exchange on high-spin projectiles [42] or multi-nucleon pickup reaction [53] may turn out to be promising tools in the arsenal of direct reactions.

Funding: Support from the U.S. Department of Energy, Office of Science, Office of Nuclear Physics, under Grant No. DE-SC0020451 is acknowledged.

Conflicts of Interest: The author declares no conflict of interest.

\section{References}

1. Brown, B. The nuclear shell model towards the drip lines. Prog. Part. Nucl. Phys. 2001, 47, 517-599. [CrossRef]

2. Otsuka, T.; Gade, A.; Sorlin, O.; Suzuki, T.; Utsuno, Y. Evolution of shell structure in exotic nuclei. Rev. Mod. Phys. 2020, 92, 015002. [CrossRef]

3. Nowacki, F.; Obertelli, A.; Poves, A. The neutron-rich edge of the nuclear landscape: Experiment and theory. Prog. Part. Nucl. Phys. 2021, 120, 103866. [CrossRef]

4. Lenzi, S.M.; Nowacki, F.; Poves, A.; Sieja, K. Island of inversion around ${ }^{64}$ Cr. Phys. Rev. C 2010, 82, 054301. [CrossRef]

5. Nowacki, F.; Poves, A.; Caurier, E.; Bounthong, B. Shape coexistence in ${ }^{78} \mathrm{Ni}$ as the portal to the fifth island of inversion. Phys. Rev. Lett. 2016, 117, 272501. [CrossRef] [PubMed]

6. Gade, A.; Liddick, S.N. Shape coexistence in neutron-rich nuclei. J. Phys. G Nucl. Part. Phys. 2016, 43, 024001. [CrossRef]

7. Brown, B.A. Islands of insight in the nuclear chart. APS Physics 2010, 3, 104. [CrossRef]

8. Hansen, P.; Tostevin, J. Direct reactions with exotic nuclei. Annu. Rev. Nucl. Part. Sci. 2003, 53, 219-261. [CrossRef]

9. Aumann, T.; Bertulani, C.A.; Ryckebusch, J. Quasifree $(p, 2 p)$ and $(p, p n)$ reactions with unstable nuclei. Phys. Rev. C 2013, 88, 064610. [CrossRef]

10. Gade, A.; Glasmacher, T. In-beam nuclear spectroscopy of bound states with fast exotic ion beams. Prog. Part. Nucl. Phys. 2008, 60, 161-224. [CrossRef]

11. Gade, A. Excitation energies in neutron-rich rare isotopes as indicators of changing shell structure. Eur. Phys. J. A 2015, 51, 118. [CrossRef]

12. Simpson, E. The Colourful Nuclide Chart. Available online: https://people.physics.anu.edu.au/ ecs103/chart/ (accessed on 1 November 2021).

13. Obertelli, A.; Delbart, A.; Anvar, S.; Audirac, L.; Authelet, G.; Baba, H.; Bruyneel, B.; Calvet, D.; Château, F.; Corsi, A.; et al. MINOS: A vertex tracker coupled to a thick liquid-hydrogen target for in-beam spectroscopy of exotic nuclei. Eur. Phys. J. A 2014, 50, 8. [CrossRef]

14. Gade, A.; Sherrill, B.M. NSCL and FRIB at Michigan State University: Nuclear science at the limits of stability. Phys. Scr. 2016, 91, 053003. [CrossRef]

15. Sakurai, H. Nuclear physics with RI Beam Factory. Front. Phys. 2018, 13, 132111. [CrossRef]

16. Paschalis, S.; Lee, I.; Macchiavelli, A.; Campbell, C.; Cromaz, M.; Gros, S.; Pavan, J.; Qian, J.; Clark, R.; Crawford, H.; et al. The performance of the Gamma-Ray Energy Tracking In-beam Nuclear Array GRETINA. Nucl. Instrum. Meth. Phys. Res. Sect. Accel. Spectrometers Detect. Assoc. Equip. 2013, 709, 44-55. [CrossRef]

17. Weisshaar, D.; Bazin, D.; Bender, P.; Campbell, C.; Recchia, F.; Bader, V.; Baugher, T.; Belarge, J.; Carpenter, M.; Crawford, H.; et al. The performance of the $\gamma$-ray tracking array GRETINA for $\gamma$-ray spectroscopy with fast beams of rare isotopes. Nucl. Instrum. Meth. Phys. Res. Sect. A Accel. Spectrometers Detect. Assoc. Equip. 2017, 847, 187-198. [CrossRef]

18. Takeuchi, S.; Motobayashi, T.; Togano, Y.; Matsushita, M.; Aoi, N.; Demichi, K.; Hasegawa, H.; Murakami, H. DALI2: A NaI(Tl) detector array for measurements of $\gamma$ rays from fast nuclei. Nucl. Instrum. Meth. Phys. Res. Sect. A Accel. Spectrometers Detect. Assoc. Equip. 2014, 763, 596-603. [CrossRef]

19. Tostevin, J. Single-nucleon knockout reactions at fragmentation beam energies. Nucl. Phys. A 2001, 682, 320-331. [CrossRef]

20. Tostevin, J. Core excitation in halo nucleus break-up. J. Phys. G Nucl. Part. Phys. 1999, 25, 735-739. [CrossRef]

21. Gade, A.; Adrich, P.; Bazin, D.; Bowen, M.D.; Brown, B.A.; Campbell, C.M.; Cook, J.M.; Glasmacher, T.; Hansen, P.G.; Hosier, K.; et al. Reduction of spectroscopic strength: Weakly-bound and strongly-bound single-particle states studied using one-nucleon knockout reactions. Phys. Rev. C 2008, 77, 044306. [CrossRef]

22. Tostevin, J.A.; Gade, A. Updated systematics of intermediate-energy single-nucleon removal cross sections. Phys. Rev. C 2021, 103, 054610. [CrossRef]

23. Bazin, D.; Brown, B.A.; Campbell, C.M.; Church, J.A.; Dinca, D.C.; Enders, J.; Gade, A.; Glasmacher, T.; Hansen, P.G.; Mueller, W.F.; et al. New direct reaction: Two-proton knockout from neutron-rich nuclei. Phys. Rev. Lett. 2003, 91, 012501. [CrossRef] [PubMed] 
24. Yoneda, K.; Obertelli, A.; Gade, A.; Bazin, D.; Brown, B.A.; Campbell, C.M.; Cook, J.M.; Cottle, P.D.; Davies, A.D.; Dinca, D.C.; et al. Two-neutron knockout from neutron-deficient ${ }^{34} \mathrm{Ar},{ }^{30} \mathrm{~S}$, and ${ }^{26} \mathrm{Si}$. Phys. Rev. C 2006, 74, 021303. [CrossRef]

25. Tostevin, J.A.; Podolyák, G.; Brown, B.A.; Hansen, P.G. Correlated two-nucleon stripping reactions. Phys. Rev. C 2004, 70, 064602. [CrossRef]

26. Tostevin, J.A.; Brown, B.A. Diffraction dissociation contributions to two-nucleon knockout reactions and the suppression of shell-model strength. Phys. Rev. C 2006, 74, 064604. [CrossRef]

27. Simpson, E.C.; Tostevin, J.A.; Bazin, D.; Gade, A. Longitudinal momentum distributions of the reaction residues following fast two-nucleon knockout reactions. Phys. Rev. C 2009, 79, 064621. [CrossRef]

28. Simpson, E.C.; Tostevin, J.A.; Bazin, D.; Brown, B.A.; Gade, A. Two-nucleon knockout spectroscopy at the limits of nuclear stability. Phys. Rev. Lett. 2009, 102, 132502. [CrossRef] [PubMed]

29. Santiago-Gonzalez, D.; Wiedenhöver, I.; Abramkina, V.; Avila, M.L.; Baugher, T.; Bazin, D.; Brown, B.A.; Cottle, P.D.; Gade, A.; Glasmacher, T.; et al. Triple configuration coexistence in ${ }^{44}$ S. Phys. Rev. C 2011, 83, 061305. [CrossRef]

30. Simpson, E.C.; Tostevin, J.A. Correlations probed in direct two-nucleon removal reactions. Phys. Rev. C 2010, 82, 044616. [CrossRef]

31. Longfellow, B.; Gade, A.; Tostevin, J.A.; Simpson, E.C.; Brown, B.A.; Magilligan, A.; Bazin, D.; Bender, P.C.; Bowry, M.; Elman, B.; et al. Two-neutron knockout as a probe of the composition of states in ${ }^{22} \mathrm{Mg}^{23} \mathrm{Al}$, and ${ }^{24} \mathrm{Si}$. Phys. Rev. C 2020, 101, 031303. [CrossRef]

32. Aumann, T.; Barbieri, C.; Bazin, D.; Bertulani, C.; Bonaccorso, A.; Dickhoff, W.; Gade, A.; Gomez-Ramos, M.; Kay, B.; Moro, A.; et al. Quenching of single-particle strength from direct reactions with stable and rare-isotope beams. Prog. Part. Nucl. Phys. 2021, 118, 103847. [CrossRef]

33. Adrich, P.; Amthor, A.M.; Bazin, D.; Bowen, M.D.; Brown, B.A.; Campbell, C.M.; Cook, J.M.; Gade, A.; Galaviz, D.; Glasmacher, T.; et al. In-beam $\gamma$-ray spectroscopy and inclusive two-proton knockout cross section measurements at $N \approx 40$. Phys. Rev. C 2008, 77, 054306. [CrossRef]

34. Hannawald, M.; Kautzsch, T.; Wöhr, A.; Walters, W.B.; Kratz, K.L.; Fedoseyev, V.N.; Mishin, V.I.; Böhmer, W.; Pfeiffer, B.; Sebastian, V.; et al. Decay of neutron-rich Mn nuclides and deformation of heavy Fe isotopes. Phys. Rev. Lett. 1999, 82, 1391-1394. [CrossRef]

35. Liddick, S.N.; Abromeit, B.; Ayres, A.; Bey, A.; Bingham, C.R.; Brown, B.A.; Cartegni, L.; Crawford, H.L.; Darby, I.G.; Grzywacz, R.; et al. Low-energy level schemes of ${ }^{66,68} \mathrm{Fe}$ and inferred proton and neutron excitations across $Z=28$ and $N=40$. Phys. Rev. C 2013, 87, 014325. [CrossRef]

36. Kotila, J.; Lenzi, S.M. Collective features of Cr and Fe isotopes. Phys. Rev. C 2014, 89, 064304. [CrossRef]

37. Gade, A.; Janssens, R.V.F.; Baugher, T.; Bazin, D.; Brown, B.A.; Carpenter, M.P.; Chiara, C.J.; Deacon, A.N.; Freeman, S.J.; Grinyer, G.F.; et al. Collectivity at $N=40$ in neutron-rich ${ }^{64}$ Cr. Phys. Rev. C 2010, 81, 051304. [CrossRef]

38. Crawford, H.L.; Clark, R.M.; Fallon, P.; Macchiavelli, A.O.; Baugher, T.; Bazin, D.; Beausang, C.W.; Berryman, J.S.; Bleuel, D.L.; Campbell, C.M.; et al. Quadrupole collectivity in neutron-rich Fe and Cr isotopes. Phys. Rev. Lett. 2013, 110, 242701. [CrossRef]

39. Rother, W.; Dewald, A.; Iwasaki, H.; Lenzi, S.M.; Starosta, K.; Bazin, D.; Baugher, T.; Brown, B.A.; Crawford, H.L.; Fransen, C.; et al. Enhanced quadrupole collectivity at $N=40$ : The case of neutron-rich Fe isotopes. Phys. Rev. Lett. 2011, $106,022502$. [CrossRef]

40. Daugas, J.M.; Matea, I.; Delaroche, J.P.; Pfützner, M.; Sawicka, M.; Becker, F.; Bélier, G.; Bingham, C.R.; Borcea, R.; Bouchez, E.; et al. $\beta$-decay measurements for $N>40 \mathrm{Mn}$ nuclei and inference of collectivity for neutron-rich Fe isotopes. Phys. Rev. C 2011, 83, 054312. [CrossRef]

41. Benzoni, G.; Morales, A.; Watanabe, H.; Nishimura, S.; Coraggio, L.; Itaco, N.; Gargano, A.; Browne, F.; Daido, R.; Doornenbal, P.; et al. Decay properties of 68,69,70 Mn: Probing collectivity up to $N=44$ in Fe isotopic chain. Phys. Lett. B 2015, 751, 107-112. [CrossRef]

42. Gade, A.; Janssens, R.V.F.; Brown, B.A.; Zegers, R.G.T.; Bazin, D.; Farris, P.; Hill, A.M.; Li, J.; Little, D.; Longfellow, B.; et al. In-beam $\gamma$-ray spectroscopy of ${ }^{68} \mathrm{Fe}$ from charge exchange on ${ }^{68} \mathrm{Co}$ projectiles. Phys. Rev. C 2021, 104, 024313. [CrossRef]

43. Santamaria, C.; Louchart, C.; Obertelli, A.; Werner, V.; Doornenbal, P.; Nowacki, F.; Authelet, G.; Baba, H.; Calvet, D.; Château, F.; et al. Extension of the $N=40$ Island of inversion towards $N=50$ : Spectroscopy of ${ }^{66} \mathrm{Cr},{ }^{70,72} \mathrm{Fe}$. Phys. Rev. Lett. 2015, 115, 192501. [CrossRef]

44. Gade, A.; Janssens, R.V.F.; Tostevin, J.A.; Bazin, D.; Belarge, J.; Bender, P.C.; Bottoni, S.; Carpenter, M.P.; Elman, B.; Freeman, S.J.; et al. Structure of ${ }^{70}$ Fe: Single-particle and collective degrees of freedom. Phys. Rev. C 2019, 99, 011301. [CrossRef]

45. Hardy, J.; Carraz, L.; Jonson, B.; Hansen, P. The essential decay of pandemonium: A demonstration of errors in complex beta-decay schemes. Phys. Lett. B 1977, 71, 307-310. [CrossRef]

46. Suchyta, S.; Liddick, S.N.; Chiara, C.J.; Walters, W.B.; Carpenter, M.P.; Crawford, H.L.; Grinyer, G.F.; Gürdal, G.; Klose, A.; McCutchan, E.A.; et al. $\beta$ and isomeric decay of ${ }^{64}$ V. Phys. Rev. C 2014, 89, 067303. [CrossRef]

47. Gade, A.; Janssens, R.V.F.; Bazin, D.; Farris, P.; Hill, A.M.; Lenzi, S.M.; Li, J.; Little, D.; Longfellow, B.; Nowacki, F.; et al. In-beam $\gamma$-ray spectroscopy of ${ }^{62,64} \mathrm{Cr}$. Phys. Rev. C 2021, 103, 014314. [CrossRef]

48. Gade, A.; Janssens, R.V.F.; Weisshaar, D.; Brown, B.A.; Lunderberg, E.; Albers, M.; Bader, V.M.; Baugher, T.; Bazin, D.; Berryman, J.S.; et al. Nuclear structure towards $N=40{ }^{60}$ Ca: In-beam $\gamma$-ray spectroscopy of ${ }^{58,60} \mathrm{Ti}$. Phys. Rev. Lett. 2014, 112, 112503. [CrossRef] 
49. Cortes, M.; Rodriguez, W.; Doornenbal, P.; Obertelli, A.; Holt, J.; Lenzi, S.; Menendez, J.; Nowacki, F.; Ogata, K.; Poves, A.; et al. Shell evolution of $N=40$ isotones towards ${ }^{60}$ Ca: First spectroscopy of ${ }^{62}$ Ti. Phys. Lett. B 2020, 800, 135071. [CrossRef]

50. Tarasov, O.B.; Ahn, D.S.; Bazin, D.; Fukuda, N.; Gade, A.; Hausmann, M.; Inabe, N.; Ishikawa, S.; Iwasa, N.; Kawata, K.; et al. Discovery of ${ }^{60} \mathrm{Ca}$ and Implications For the Stability of ${ }^{70} \mathrm{Ca}$. Phys. Rev. Lett. 2018, 121, 022501. [CrossRef]

51. Coraggio, L.; Covello, A.; Gargano, A.; Itaco, N. Realistic shell-model calculations for isotopic chains "north-east" of ${ }^{48} \mathrm{Ca}$ in the $(N, Z)$ plane. Phys. Rev. C 2014, 89, 024319. [CrossRef]

52. Liu, X.; Liu, Z.; Ding, B.; Doornenbal, P.; Obertelli, A.; Lenzi, S.; Walker, P.; Chung, L.; Linh, B.; Authelet, G.; et al. Spectroscopy of 2565,67Mn: Strong coupling in the $N=40$ island of inversion. Phys. Lett. B 2018, 784, 392-396. [CrossRef]

53. Gade, A.; Weisshaar, D.; Brown, B.; Tostevin, J.; Bazin, D.; Brown, K.; Charity, R.; Farris, P.; Hill, A.; Li, J.; et al. In-beam $\gamma$-ray spectroscopy at the proton dripline: ${ }^{40}$ Sc. Phys. Lett. B 2020, 808, 135637. [CrossRef] 